\title{
Novel Method of Predicting the Optimal Atrioventricular Delay in Patients With Complete AV Block, Normal Left Ventricular Function and an Implanted DDD Pacemaker
}

\author{
Yuko Miki, MD; Toshiyuki Ishikawa, MD; Kohei Matsushita, MD; Youhei Yamakawa, MD; \\ Katsumi Matsumoto, MD; Shinichi Sumita, MD; Kazuaki Uchino, MD; \\ Kazuo Kimura, MD; Satoshi Umemura, MD
}

\begin{abstract}
Background: The optimal atrioventricular (AV) delay setting is important for achieving optimal AV synchrony in patients with an implanted DDD pacemaker. Using pulsed Doppler echocardiography is the most common method of predicting the optimal AV delay, but it is a complicated and time-consuming method. Therefore, an automatic optimizing function of the AV delay at different atrial rates is desirable for achieving a favorable hemodynamic state. This study aimed to predict the optimal AV delay using phonocardiography.

Methods and Results: The amplitude of the first heart sound (S1) recorded on the phonocardiogram was measured with different AV delays in 6 patents with complete AV block, normal left ventricular function and an implanted DDD pacemaker. The correlation between the amplitude of S1 and the length of the AV delay was a cubic curve $\left(y=974.15 x^{3}-23.084 x^{2}-8.0074 x+0.7495, R^{2}=0.9511\right)$. The length of the AV delay at the inflection point of the curve showed a significant positive correlation with the optimal AV delay determined by pulsed Doppler echocardiography $(\mathrm{R}=0.9254, \mathrm{P}<0.01)$.

Conclusions: This study demonstrated a novel simple method of predicting the optimal AV delay using phonocardiography. (Circ J 2009; 73: 654-657)
\end{abstract}

Key Words: Atrioventricular delay; Echocardiography; Pacemaker; Phonocardiography

I is important to set the optimal atrioventricular (AV) delay to achieve optimal AV synchrony in patients with an implanted DDD pacemaker-6 The optimal $\mathrm{AV}$ delay allows completion of end-diastolic filling flow prior to ventricular contraction, providing the longest diastolic filling time, consequently increasing cardiac output and reducing the pulmonary wedge pressure. To determine the optimal AV delay, pulsed Doppler echocardiography is commonly used, and our method was reported previously5,7-13 However, echocardiography has several problems. The examination is time-consuming, and the optimal $\mathrm{AV}$ delay varies from patient to patient and with the patient's heart rate during exercise, sleep and other activities. Therefore, although the optimal AV delay is set using echocardiography only once, that value may not be adequate in the patient's daily life. If a more simple method of assessing the optimal AV delay was available, the delay could be set more frequently. Therefore, the aim of this study was to predict the optimal AV delay by a novel method using phonocardiography.

(Received April 16, 2008; revised manuscript received November 14, 2008; accepted December 7, 2008; released online February 26, 2009) Department of Medical Science and Cardiorenal Medicine, Yokohama City University School of Medicine, Yokohama, Japan

Name of grant.

Mailing address: Yuko Miki, MD, Department of Medical Science and Cardiorenal Medicine, Yokohama City University School of Medicine, 3-9 Fukuura, Kanazawa-ku, Yokohama 236-0004, Japan All rights are reserved to the Japanese Circulation Society. For permissions, please e-mail: cj@j-circ.or.jp

\section{Methods}

\section{Study Population}

We included 6 patients (mean age 72.7 \pm 5.7 [SD] years, 3 males) with complete AV block, normal left ventricular (LV) function and an implanted DDD pacemaker (Table). Informed consent was given by each participant before enrollment in this study.

\section{Study Protocol}

The amplitude of the first heart sound (S1) was recorded on a phonocardiogram (PCG) during AV sequential pacing. Transmitral flow and the time velocity integral (TVI) of the LV outflow tract (LVOT) were measured by pulsed Doppler echocardiography at the same time. The AV delay was set from 50 to $250 \mathrm{~ms}$ in a stepwise fashion at 20 - or $25-\mathrm{ms}$ increments. The pacing rate was fixed at $70-80$ beats $/ \mathrm{min}$ to maintain atrial and ventricular pacing. All patients were examined at rest in the left lateral decubitus position, at the end of each 5-min period of AV sequential pacing.

\section{Measurement of S1}

An acceleration-type PCG microphone (MA-250, Fukuda Denshi, Tokyo, Japan) was positioned at the cardiac apex and connected to an echocardiograph. Phonocardiograms were filtered from 20 to $600 \mathrm{~Hz}$ and recorded on the echocardiograph. The S1 amplitude from peak to peak was measured. The acceleration-type PCG microphone converts the vibration of chest by the heartbeat to electrical signals according to acceleration. There was little PCG noises and 

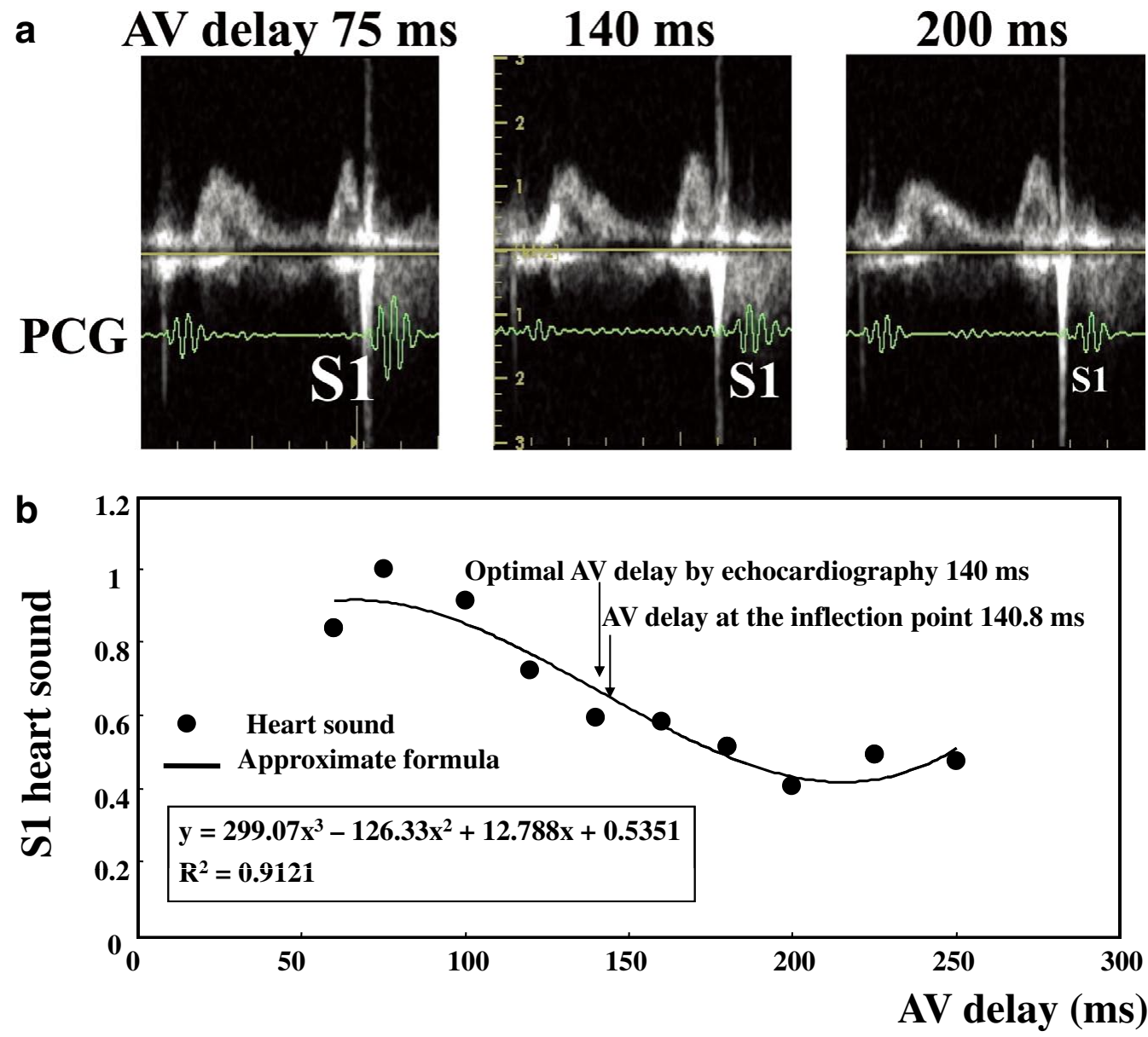

Figure 1. Representative case (patient no 3. in Table). (a) The amplitude of the first heart sound (S1) varies with the atrioventricular (AV) delay. (b) The correlation between the S1 amplitude and AV delay is a cubic curve. The AV delay at the inflection point is $140.8 \mathrm{~ms}$ and the predicted optimal AV delay using echocardiography is $140 \mathrm{~ms}$. PCG, phonocardiogram.

Table. Patients' Characteristics and Results

\begin{tabular}{ccccccc}
\hline Patient no. & Gender & Age (years) & $\begin{array}{c}\text { Ejection } \\
\text { fraction }(\%)\end{array}$ & CTR $(\%)$ & $\begin{array}{c}\text { Optimal AV delay } \\
\text { by UCG (ms) }\end{array}$ & $\begin{array}{c}\text { AV delay at } \\
\text { inflection point (ms) }\end{array}$ \\
\hline 1 & F & 68 & 60.0 & 59.6 & 150 & 168.5 \\
2 & M & 75 & 56.8 & 50.3 & 130 & 150.5 \\
3 & M & 80 & 69.9 & 43.7 & 140 & 140.8 \\
4 & M & 64 & 58.8 & 43.9 & 140 & 154.8 \\
5 & F & 75 & 60.0 & 49.6 & 120 & 111 \\
6 & F & 74 & 62.1 & 50.9 & 150 & 166.7 \\
Mean & & 72.7 & 61.3 & 49.7 & 138.3 & 148.7 \\
SD & & 5.7 & 4.6 & 5.8 & 11.7 & 21.1 \\
\hline
\end{tabular}

CTR, cardiac resynchronization therapy; AV, atrioventricular; UCG, ultrasonic cardiography.

the S1 amplitude could be measured easily without using a sound room.

\section{Analysis of S1 Characteristics}

The S1 amplitude and the LVOT TVI at each AV delay were determined by averaging 3 cardiac cycles and are expressed as relative values to the peak values. The average S1 amplitude for each AV delay vs AV delay was plotted, and the relationship between S1 amplitude and the optimal AV delay predicted by our method using pulsed Doppler echocardiography was analyzed. Measured variables were expressed as mean $\pm \mathrm{SD}$.

\section{Statistical Analysis}

Statistical analyses were performed by paired Student's t-test and Pearson's correlation coefficient analysis. Values of $\mathrm{P}<0.05$ were considered to be statistically significant.

\section{Results}

One representative case (patient no. 3) is shown in Figure 1. The patient's characteristics and results are sum- 


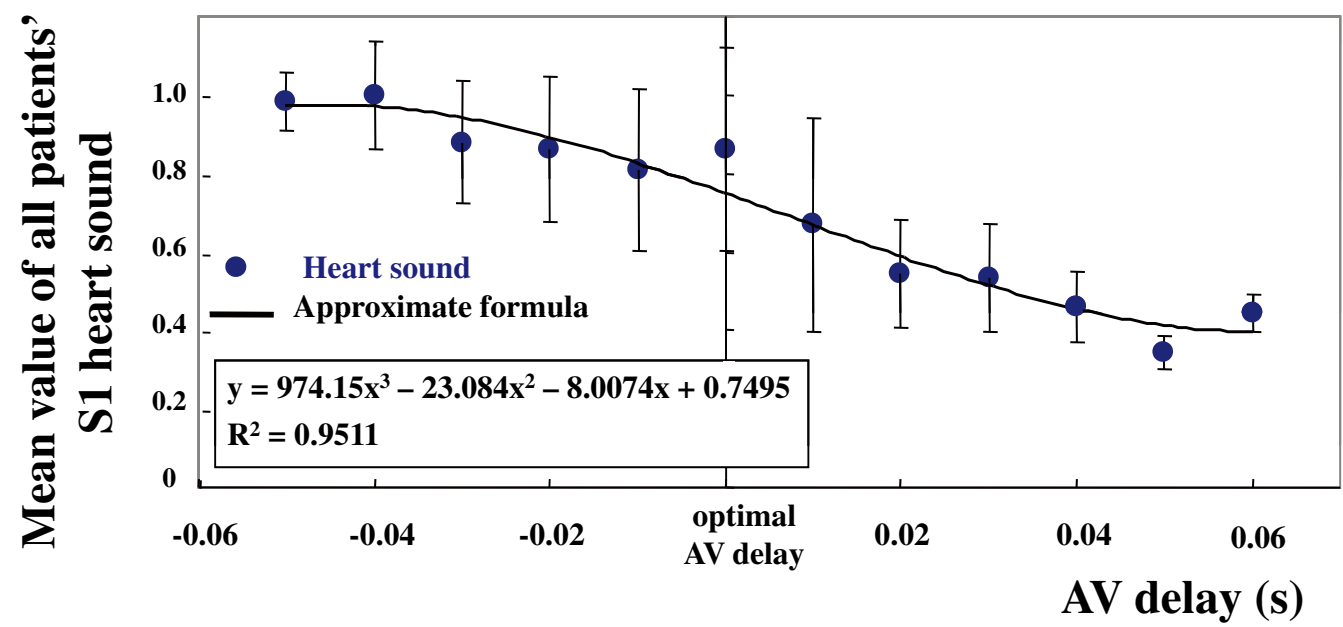

Figure 2. Correlation between the first heart sound (S1) and the atrioventricular (AV) delay is a cubic curve (y= $\left.974.15 x^{3}-23.084 x^{2}-8.0074 x+0.7495, R^{2}=0.9511\right)$.

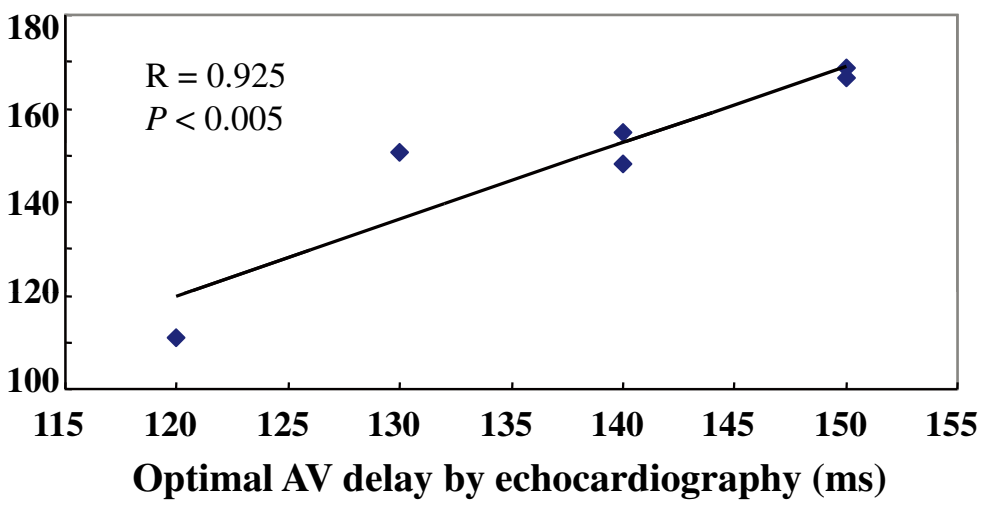

Figure 3. Atrioventricular (AV) delay at the inflection point of the cubic curve shows a significant positive correlation with the optimal AV delay determined by echocardiography $(\mathrm{R}=0.925, \mathrm{P}<0.005)$. marized in Table. The amplitude of S1 changed according to the change in AV delay. When the AV delay was shortened, the amplitude of S1 gradually increased and reached a plateau. The correlation between the amplitude of S1 and the length of AV delay showed a cubic curve (Figure 2) $\left(y=974.15 x^{3}-23.084 x^{2}-8.0074 x+0.7495, R^{2}=0.9511\right)$. The length of the AV delay at the inflection point of this cubic curve (AVDI) showed a significant positive correlation with the optimal AV delay determined by echocardiography (OAVDE) $(\mathrm{R}=0.925, \mathrm{P}<0.005)$ (Figure 3). The average AVDI was $148.7 \pm 21.1 \mathrm{~ms}$, and the average OAVDE was $138.3 \pm 11.7 \mathrm{~ms}$ (average AVDI slightly longer than the average OAVDE). The peak of TVI at the LVOT was not determined because TVI was unchanged at different AV delays ranging from 50 to $250 \mathrm{~ms}$.

\section{Discussion}

Previously we reported that the optimal AV delay could be predicted as the AV delay at which the QT interval was maximal.14-17 In the present study, we observed that the correlation between the amplitude of S1 and the length of the AV delay was represented as a cubic curve. Furthermore, the AV delay at the inflection point of this cubic curve showed a significant positive correlation with the optimal AV delay determined by echocardiography
$(\mathrm{R}=0.925, \mathrm{P}<0.005)$. The reason why the correlation between the amplitude of $\mathrm{S} 1$ and the AV delay is a cubic curve is unclear. When the atrial pressure decreases gradually after atrial contraction, the AV pressure gradient is reversed in the end-diastolic phase. The reversed AV pressure gradient may be important for mitral valve closure, but it is not sufficient to bring about complete closure of the mitral valve without ventricular contraction, 18,19 which is required for complete closure of the mitral valve. With the setting of a long AV delay, mitral valve closure is incomplete, because ventricular contraction has not yet started. Complete closure of the mitral valve is achieved by ventricular contraction with the half-open mitral valve and the amplitude of S1 is decreased with the setting of a long AV delay. When the AV delay is shortened, the amplitude of $\mathrm{S} 1$ increases gradually. When the AV delay is set extremely short, complete closure of the mitral valve is achieved in a burst without a stop against the atrial kick, and atrial contraction is interrupted. The amplitude of S1 will decrease with an extremely short AV delay setting. In this study, all patients had normal LV function and the length of the AV delay had an insignificant effect on TVI at the LVOT. In patients with complete AV block and an implanted DDD pacemaker, Ritter et al reported a highly significant difference between the optimal AV delay obtained by echocardiography and that obtained by the peak endocardial accel- 
eration (PEA) method, which corresponds to the 'knee' of the PEA curve vs the AV delay?20,21 PEA is detected by a ventricular lead equipped with a micro-accelerometer. Various reports have shown that PEA is synchronous with S1. If a pacemaker equipped with an acoustic wave sensor could be developed, automatic AV delay optimization using PCG at different atrial rates might be achieved. An activity sensor is a type of vibration sensor, and might be used for this purpose, obviating the need for a specially designed lead such as with the PEA method.

Additionally, AV delay optimization is important in patients with severely impaired cardiac function, especially in cardiac resynchronization therapy (CRT). Further examination of whether this PCG method of predicting the optimal AV delay is also useful in patients undergoing CRT will be required.

\section{Conclusions}

The present study demonstrated a novel simple method of predicting optimal AV delay using phonocardiography.

\section{References}

1. Hochleitner M, Hörtnagl H, Ng CK, Hörtnagl H, Gschnitzer F, Zechmann W. Usefulness of physiologic dual chamber pacing in drug-resistant idiopathic dilated cardiomyopathy. Am J Cardiol 1990; 66: $198-202$

2. Auricchio A, Sommariva L, Salo RW, Scafuri A, Chiariello L. Improvement of cardiac function in patients with severe congestive heart failure and coronary artery disease by dual chamber pacing with shortened AV delay. Pacing Clin Electrophysiol 1993; 16: 2034 2043.

3. Nishimura RA, Hayes DL, Holmes DR Jr, Tajik AJ. Mechanism of hemodynamic improvement by dualchamber pacing for severe left ventricular dysfunction: An acute Doppler and catheterization hemodynamic study. J Am Coll Cardiol 1995; 25: 281-288.

4. Ritter P, Dib JC, Mahaux V, Lelieve T, Soyeur D, Lavergne T, et al. New method for determining the optimal atrioventricular delay in paced in DDD mode for complete atrio ventricular block (abstract). Pacing Clin Electrophysiol 1995; 18: 855.

5. Ishikawa T, Sumita S, Kimura K, Kikuchi M, Kosuge M, Kuji N, et al. Prediction of optimal atrioventricular delay in patients with implanted DDD pacemakers. Pacing Clin Electrophysiol 1999; 22: $1365-1371$.

6. Ritter P, Daubert C, Mabo P, Descaves C, Gouffault J. Haemodynamic benefit of a rate adapted A-V delay in dual chamber pacing. Eur Heart J 1989; 10: 636-646.

7. Ishikawa T, Usui T, Kashiwagi M, Kimura K, Yoshimura H, Sano T, et al. Contribution of end-diastolic mitral regurgitation to the B-B' step formation on M-mode echocardiography. J Appl Cardiol 1991; 6: $163-168$.
8. Ishikawa T, Kimura K, Nihei T, Usui T, Kashiwagi M, Ishii M, et al. Relationship between diastolic mitral regurgitation and $\mathrm{PQ}$ interval or cardiac function in patients implanted with DDD pacemakers. Pacing Clin Electrophysiol 1991; 14: 1797-1802.

9. Ishikawa T, Kimura K, Miyazaki N, Tochikubo O, Usui T, Kashiwagi M, et al. Diastolic mitral regurgitation in patients with firstdegree atrioventricular block. Pacing Clin Electrophysiol 1992; 15: $1927-1931$.

10. Ishikawa T, Sumita S, Kimura K, Ebina T, Kuji N, Miyazaki N, et al. PQ interval and diastolic mitral regurgitation in patients with sick sinus syndrome. Eur J Cardiac Pacing Electrophysiol 1994; 4: $170-175$.

11. Ishikawa T, Sumita S, Kimura K, Kuji N, Nakayama R, Nagura T, et al. Critical PQ interval for the appearance of diastolic mitral regurgitation and optimal PQ interval in patients implanted with DDD pacemakers. Pacing Clin Electrophysiol 1994; 17: 1989-1994.

12. Ishikawa T, Sumita S, Kikuchi M, Satho T, Terada K, Kuji N, et al. Diastolic mitral regurgitation when the heart rate is normalized by ventricular pacing. Eur J Cardiac Pacing Electrophysiol 1996; 6: $23-27$.

13. Ishikawa T, Sumita S, Kimura K, Kimura M, Kuji N, Endo T, et al. Optimal atrioventricular setting and diastolic mitral regurgitation in patients implanted with DDD pacemakers. Eur J Cardiac Pacing Electrophysiol 1996; 6: 159-163.

14. Ishikawa T, Sugano T, Sumita S, Kosuge M, Kobayashi I, Kobayashi T, et al. Changes in evoked QT intervals according to variations in atrioventricular delay and cardiac function in patients with implanted QT-driven DDDR pacemakers. Circ J 2003; 67: $515-518$.

15. Ishikawa T, Sugano T, Sumita S, Kimura K, Kikuchi M, Kosuge M, et al. Relationship between atrioventricular delay, QT interval and cardiac function in patients with implanted DDD pacemakers. Europace 1999; 1: 192-196.

16. Ishikawa T, Suganao T, Sumita S, Nakagawa T, Hanada M, Kosuge $\mathrm{M}$, et al. Optimal atrioventricular delay setting determined by evoked QT interval in patients with implanted stimulus-T-driven DDDR pacemakers. Europace 2001; 3: 46-51.

17. Ishikawa T, Sugano T, Sumita S, Toda N, Kosuge M, Kobayashi I, et al. Optimal atrioventricular delay setting determined by QT sensor of implanted DDDR pacemaker. Pacing Clin Electrophysiol 2002; 25: $195-200$.

18. Rutishauser W, Wirz P, Gander M, Lüthy E. Atriogenic diastolic reflux in patients with atrioventricular block. Circulation 1966; 34: 807-817.

19. Nolan SP, Dixon SH Jr, Fisher RD, Morrow AG. The influence of atrial contraction and mitral valve mechanism on ventricular filling: A study of instantaneous mitral valve flow in vivo. Am Heart J 1969; 77: 787-791.

20. Ritter P, Padeletti L, Gillio-Meina L, Gaggini G. Determination of the optimal atrioventricular delay in DDD pacing: Comparison between echo and peak endocardial acceleration measurements. Europace 1999; 1: 126-130.

21. Dupuis JM, Kobeissi A, Vitali L, Gaggini G, Merheb M, Rouleau F, et al. Programming optimal atrioventricular delay in dual chamber pacing using peak endocardial acceleration: Comparison with a standard echocardiographic procedure. Pacing Clin Electrophysiol 2003; 26: $210-213$. 